\title{
Radio Emission from SNe and Young SNRs
}

\author{
Kurt W. Weiler ${ }^{1}$, Nino Panagia ${ }^{2}$, Marcos J. Montes ${ }^{1}$, Schuyler \\ D. Van Dyk ${ }^{3}$, Richard A. Sramek ${ }^{4}$, and Christina K. Lacey ${ }^{5}$ \\ ${ }^{1}$ NRL, Code 7213, Washington, DC 20375-5320, USA \\ ${ }^{2}$ STScI, 3700 San Martin Drive, Baltimore, MD 21218, USA \& Ap. Div., Space Sci. Dept. of \\ ESA \\ ${ }^{3}$ IPAC/Caltech, Mail Code 100-22, Pasadena, CA 91125, USA \\ ${ }^{4}$ P.O. Box 0, NRAO, Socorro, NM 87801, USA \\ ${ }^{5}$ Univ. of South Carolina, Dept. of Phys. and Astron., Columbia, SC 29208, USA
}

\begin{abstract}
Study of radio supernovae (RSNe), the earliest stages of supernova remnant (SNR) formation, over the past 20 years includes two dozen detected objects and more than 100 upper limits. From this work we are able to identify classes of radio properties, demonstrate conformance to and deviations from existing models, estimate the density and structure of the circumstellar material and, by inference, the evolution of the presupernova stellar wind, and reveal the last stages of stellar evolution before explosion. It is also possible to detect ionized hydrogen along the line of sight, to demonstrate binary properties of the stellar system, and to show clumpiness of the circumstellar material. More speculatively, it may be possible to provide distance estimates to RSNe.
\end{abstract}

\section{INTRODUCTION}

A series of papers published over the past 20 years on radio supernovae (RSNe), the earliest stages of supernova remnant (SNR) formation, has established the radio detection and, in a number of cases, radio evolution for approximately two dozen objects: 3 Type Ib supernovae (SNe), 5 Type Ic SNe, and the rest Type II SNe. A much larger number of more than 100 additional SNe have low radio upper limits.

In this extensive study of the radio emission from SNe and young SNRs, several effects have been noted: 1) Type Ia SNe are not radio emitters to the detection limit of the Very Large Array ${ }^{1}$ (VLA); 2) Type Ib and Ic SNe are radio luminous

1) The VLA telescope of the National Radio Astronomy Observatory is operated by Associated Universities, Inc. under a cooperative agreement with the National Science Foundation.

CP565, Young Supernova Remnants: Eleventh Astrophysics Conf., edited by S. S. Holt and U. Hwang (1) 2001 American Institute of Physics 0-7354-0001-6/01/\$18.00 
with steep spectral indices (generally $\alpha<-1 ; S \propto \nu^{+\alpha}$ ) and a fast turn-on/turnoff, usually peaking at $6 \mathrm{~cm}$ near or before optical maximum; and 3) Type II SNe show a range of radio luminosities with flatter spectral indices (generally $\alpha>-1$ ) and a relatively slow turn-on/turn-off, usually peaking at $6 \mathrm{~cm}$ significantly after optical maximum. Type Ib and Ic may be fairly homogeneous in some of their radio properties while Type II, as in the optical, are quite diverse.

There are a large number of physical properties of SNe which we can determine from radio observations. VLBI imaging shows the symmetry of the explosion and the local circumstellar medium (CSM), estimates the speed and deceleration of the SN blastwave propagating outward from the explosion and, with assumptions of symmetry and optical line/radio-sphere velocities, allows independent distance estimates to be made (see, e.g., [1,2]). Measurements of the multi-frequency radio light curves and their evolution with time show the density and structure of the CSM, evidence for possible binary companions, clumpiness or filamentation in the presupernova wind, mass-loss rates and changes therein for the presupernova stellar system and, through stellar evolution models, estimates of the ZAMS presupernova stellar mass and the stages through which the star passed on its way to explosion. It has also been proposed by Weiler, et al. [3] that the time from explosion to $6 \mathrm{~cm}$ radio maximum may be an indicator of the radio luminosity and thus an independent distance indicator for Type II SNe and that Type Ib and Ic SNe may be approximate radio standard candles at $6 \mathrm{~cm}$ radio peak flux density.

\section{MODELS}

All known RSNe appear to share common properties of: 1) nonthermal synchrotron emission with high brightness temperature; 2) a decrease in absorption with time, resulting in a smooth, rapid turn-on first at shorter wavelengths and later at longer wavelengths; 3) a power-law decline of the flux density with time at each wavelength after maximum flux density (optical depth $\sim 1$ ) is reached at that wavelength; and 4) a final, asymptotic approach of spectral index $\alpha\left(S \propto \nu^{+\alpha}\right)$ to an optically thin, nonthermal, constant negative value $[4,5]$. Chevalier $[6,7]$ has proposed that the relativistic electrons and enhanced magnetic field necessary for synchrotron emission arise from the SN blastwave interacting with a relatively high density CSM which has been ionized and heated by the initial UV/X-ray flash. This CSM is presumed to have been established by a constant mass-loss $(M)$ rate, constant velocity $(w)$ wind $\left(i . e ., \rho \propto r^{-2}\right.$ ) from a massive stellar progenitor or companion. This ionized CSM is the source of some or all of the initial absorption although Chevalier [8] has proposed that synchrotron self-absorption (SSA) may play a role in some objects.

A rapid rise in the observed radio flux density results from a decrease in these absorption processes as the radio emitting region expands and the absorption processes consequently decrease. Weiler, et al. [5] have suggested that this CSM can be "clumpy" or "filamentary," leading to a slower radio turn-on, and Montes, et 
al. [9] have proposed the possible presence of a distant ionized medium along the line-of-sight which is time independent and can cause a spectral turn-over at low radio frequencies. In addition to clumps or filaments, the CSM may be structured with significant density irregularities such as rings, disks, shells, or gradients.

\section{Parameterized Radio Light Curves}

Following $[4,5,9]$, we adopt a parameterized model (N.B.: The notation is extended and rationalized here from previous publications. However, the "old" notation of $\tau, \tau^{\prime}$, and $\tau^{\prime \prime}$, which has been used previously, is noted, where appropriate, for continuity.):

$$
\begin{gathered}
S(\mathrm{mJy})=K_{1}\left(\frac{\nu}{5 \mathrm{GHz}}\right)^{\alpha}\left(\frac{t-t_{0}}{1 \text { day }}\right)^{\beta} e^{-\tau_{\text {external }}}\left(\frac{1-e^{-\tau_{\mathrm{CSM}_{\mathrm{clumps}}}}}{\tau_{\mathrm{CSM}_{\mathrm{clumps}}}}\right)\left(\frac{1-e^{-\tau_{\text {internal }}}}{\tau_{\text {internal }}}\right) \\
\tau_{\text {external }}=\tau_{\mathrm{CSM}_{\text {uniform }}}+\tau_{\text {distant }}=\tau+\tau^{\prime \prime} \\
\tau_{\mathrm{CSM}_{\text {uniform }}}=\tau=K_{2}\left(\frac{\nu}{5 \mathrm{GHz}}\right)^{-2.1}\left(\frac{t-t_{0}}{1 \text { day }}\right)^{\delta} \\
\tau_{\text {distant }}=\tau^{\prime \prime}=K_{4}\left(\frac{\nu}{5 \mathrm{GHz}}\right)^{-2.1} \\
\tau_{\mathrm{CSM}_{\text {clumps }}}=\tau^{\prime}=K_{3}\left(\frac{\nu}{5 \mathrm{GHz}}\right)^{-2.1}\left(\frac{t-t_{0}}{1 \text { day }}\right)^{\delta^{\prime}}
\end{gathered}
$$

with $K_{1}, K_{2}, K_{3}$, and $K_{4}$ corresponding, formally, to the flux density $\left(K_{1}\right)$, uniform $\left(K_{2}, K_{4}\right)$ and clumpy or filamentary $\left(K_{3}\right)$ absorption, at $5 \mathrm{GHz}$ one day after the explosion date $t_{0}$. The terms $\tau_{\mathrm{CSM}_{\text {uniform }}}$ and $\tau_{\mathrm{CSM}_{\text {clumps }}}$ describe the attenuation of local, uniform CSM and clumpy CSM that are near enough to the SN progenitor that they are altered by the rapidly expanding SN blastwave. $\tau_{\mathrm{CSM}_{\text {uniform }}}$ is produced by an ionized medium that uniformly covers the emitting source ("uniform external absorption"), and the $\left(1-e^{-\tau_{\mathrm{CSM}} \mathrm{clumps}}\right) \tau_{\mathrm{CSM}_{\mathrm{clumpS}}}^{-1}$ term describes the attenuation produced by an inhomogeneous medium with optical depths distributed, with equal probability, between 0 and $\tau_{\mathrm{CSM}_{\text {clumps }}}$ ("clumpy absorption"; see Natta \& Panagia [10] for a more detailed discussion of attenuation in inhomogeneous media). The $\tau_{\text {distant }}$ term describes the attenuation produced by a homogeneous medium which uniformly covers the source but is so far from the SN progenitor that it is not affected by the expanding SN blastwave and is constant in time. All absorbing 
media are assumed to be purely thermal, singly ionized gas which absorbs via f-f transitions with frequency dependence $\nu^{-2.1}$. The parameters $\delta$ and $\delta^{\prime}$ describe the time dependence of the optical depths for the local uniform and clumpy or filamentary media, respectively.

Since it is physically realistic and may be needed in some RSNe where radio observations have been obtained at early times and high frequencies, we have also included in Eq. 1 the possibility for an internal absorption term. This internal absorption $\left(\tau_{\text {internal }}\right)$ term may consist of two parts - SSA $\left(\tau_{\text {internal }}\right.$ SSA $)$, and mixed, thermal f-f absorption/non-thermal emission $\left(\tau_{\text {internalff }}\right)$ :

$$
\begin{gathered}
\tau_{\text {internal }}=\tau_{\text {internal }_{\text {sa }}}+\tau_{\text {internal }_{\text {ff }}} \\
\tau_{\text {internal }_{\text {ssa }}}=K_{5}\left(\frac{\nu}{5 \mathrm{GHz}}\right)^{\alpha-2.5}\left(\frac{t-t_{0}}{1 \text { day }}\right)^{\delta^{\prime \prime}} \\
\tau_{\text {internal }_{\text {ff }}}=K_{6}\left(\frac{\nu}{5 \mathrm{GHz}}\right)^{-2.1}\left(\frac{t-t_{0}}{1 \text { day }}\right)^{\delta^{\prime \prime}}
\end{gathered}
$$

with $K_{5}$ and $K_{6}$ corresponding, formally, to the internal, non-thermal $\left(\nu^{\alpha-2.5}\right)$ SSA $\left(K_{5}\right)$ and the internal, mixed with nonthermal emission, thermal $\left(\nu^{-2.1}\right)$ free-free absorption $\left(K_{6}\right)$, respectively, at $5 \mathrm{GHz}$ one day after the explosion date $t_{0}$. The parameters $\delta^{\prime \prime}$ and $\delta^{\prime \prime \prime}$ describe the time dependence of the optical depths for the SSA and f-f internal absorption components, respectively.

A cartoon of the expected structure of an SN and its surrounding media is presented in Fig. 1 (see also, Lozinskaya [11]). The radio emission is expected to arise near the blastwave [12].

\section{RESULTS}

The success of the basic parameterization and model description can be seen in the relatively good correspondence between the model fits and the data for all three subtypes of RSNe, e.g., Type Ib SN1983N (Fig. 2a, [13]), Type Ic SN1990B (Fig. 2b, [14]), Type II SN1979C (Fig. 3a, [15]) and Type II SN1980K (Fig. 3b, [16]). (Note that after day $\sim 4000$, the evolution of the radio emission from both SN1979C and SN1980K deviates from the expected model evolution and that SN1979C shows a sinusoidal modulation in its flux density $[17-19]$.)

\section{Mass-Loss Rate}

From the Chevalier [6,7] model, the turn-on of the radio emission for RSNe provides a measure of the presupernova mass-loss rate to wind velocity ratio $(\dot{M} / w)$. 


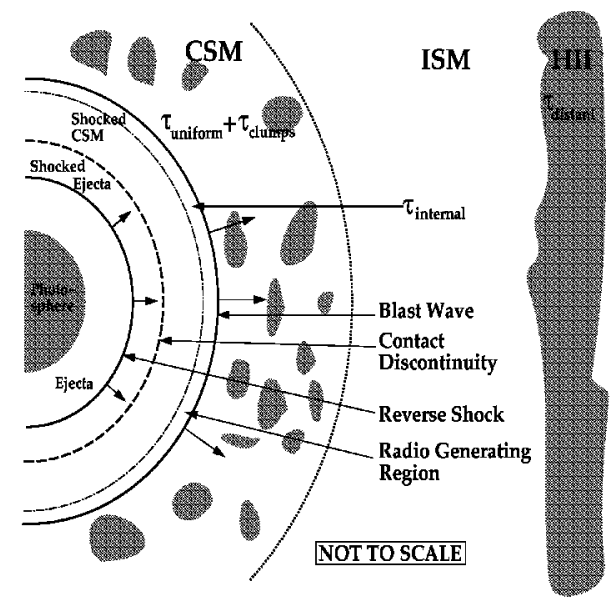

FIGURE 1. Cartoon, not to scale, of the SN and its shocks along with the stellar wind established CSM and more distant ionized material. The radio emission is thought to arise near the blastwave. The expected locations of the several absorbing terms in Eqs. $1-8$ are illustrated.
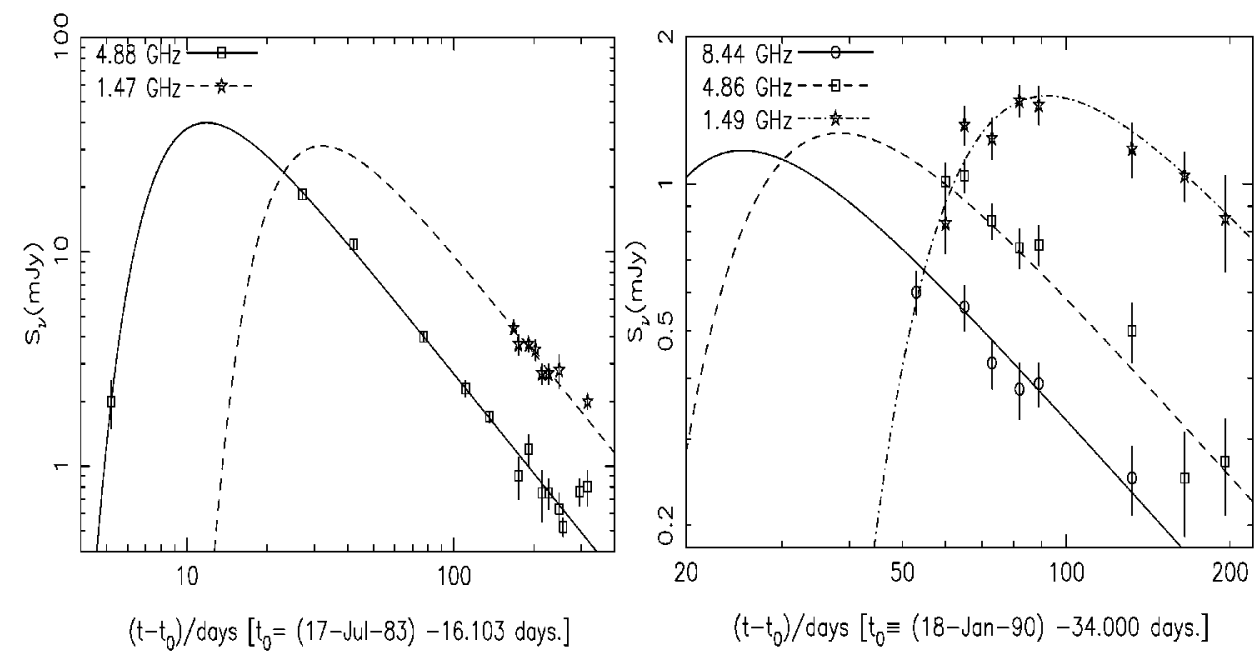

FIGURE 2. Type Ib SN1983N (left, 2a, [13]) at $6 \mathrm{~cm}(4.9 \mathrm{GHz} ;$ squares, solid line) and $20 \mathrm{~cm}$ (1.5 GHz; stars, dashed line) and Type Ic SN1990B (right, 2b, [14]) at $3.4 \mathrm{~cm}(8.4 \mathrm{GHz}$; circles, solid line), $6 \mathrm{~cm}(4.9 \mathrm{GHz}$; squares, dashed line), and $20 \mathrm{~cm}(1.5 \mathrm{GHz} ;$ stars, dash-dot line). 

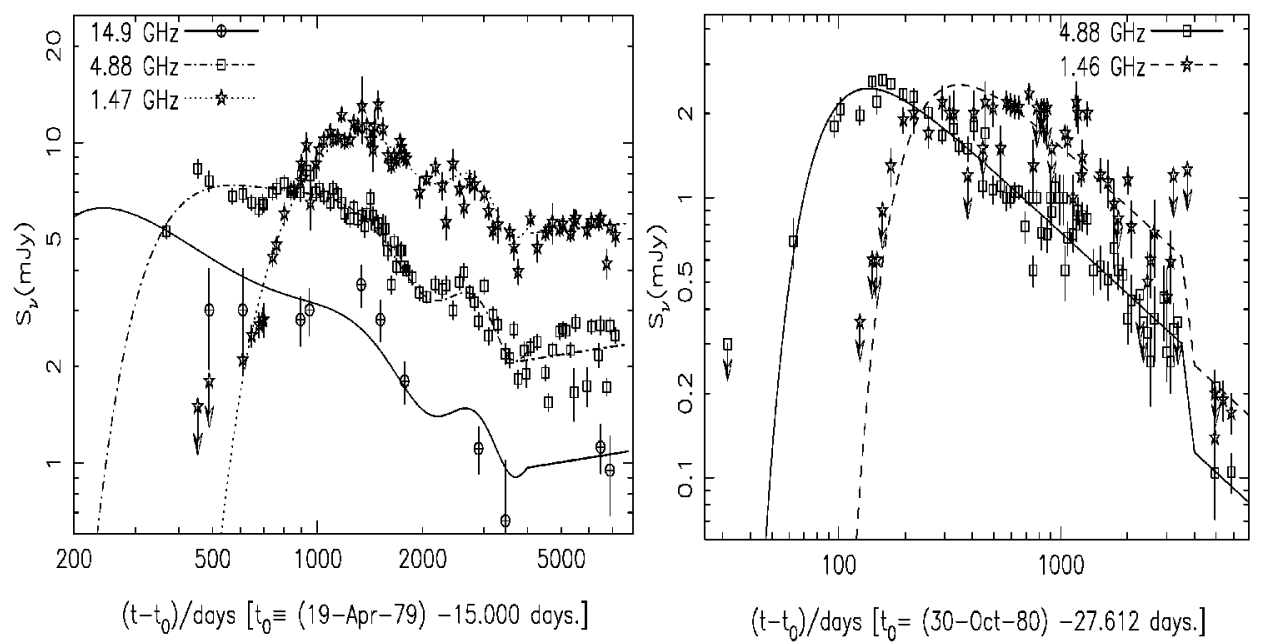

FIGURE 3. Type II SN1979C (left, 3a, [15]) at $2 \mathrm{~cm}(14.9 \mathrm{GHz}$; crossed circles, solid line), 6 $\mathrm{cm}(4.9 \mathrm{GHz}$; squares, dash-dot line), and $20 \mathrm{~cm}(1.5 \mathrm{GHz}$; stars, dotted line) (For discussion of the increase in the radio flux density after day $\sim 4000$ and the sinusoidal modulation of the radio emission, see [15,17-19]) and Type II SN1980K (right, 3b, [16]) at $6 \mathrm{~cm}(4.9 \mathrm{GHz}$; squares, solid line), and $20 \mathrm{~cm}(1.5 \mathrm{GHz}$; stars, dashed line) (For discussion of the sharp drop in flux density after day $\sim 4000$, see [16].).

For the case in which the external absorption is entirely due to a uniform medium, using the formulation of Weiler, et al. [4] Eq. 16, we can write

$$
\begin{aligned}
\frac{\dot{M}\left(\mathrm{M}_{\odot} \mathrm{yr}^{-1}\right)}{\left(w / 10 \mathrm{~km} \mathrm{~s}^{-1}\right)}= & 3 \times 10^{-6} \tau_{C S M_{\text {uniform }}^{0.5}} m^{-1.5}\left(\frac{v_{i}}{10^{4} \mathrm{~km} \mathrm{~s}^{-1}}\right)^{1.5} \times \\
& \left(\frac{t_{i}}{45 \text { days }}\right)^{1.5}\left(\frac{t}{t_{i}}\right)^{1.5 m}\left(\frac{T}{10^{4} \mathrm{~K}}\right)^{0.68}
\end{aligned}
$$

Since the appearance of optical lines for measuring SN ejecta velocities is often delayed, we arbitrarily take $t_{i}=45$ days. Because our observations have shown that, generally, $0.8 \leq m \leq 1.0(m=-\delta / 3,[4])$ and, from Eq. $9, \dot{M} \propto t_{i}^{1.5(1-m)}$, the dependence of the calculated mass-loss rate on the date $t_{i}$ of the initial ejecta velocity measurement is weak, $\dot{M} \propto t_{i}^{<0.3}$. Thus, we can take the best optical or VLBI velocity measurements available without worrying about the deviation of their exact measurement epoch from the assumed 45 days after explosion. For convenience, and because many SN measurements indicate velocities of $\sim 10,000$ $\mathrm{km} \mathrm{s}^{-1}$, we take $v_{i}=10,000 \mathrm{~km} \mathrm{~s}^{-1}$. We also adopt values of $T=20,000 \mathrm{~K}$, $w=10 \mathrm{~km} \mathrm{~s}^{-1}$ (which is appropriate for a RSG wind) and, generally from our best fits to SN radio data, $t=\left(t_{6 \mathrm{~cm} \text { peak }}-t_{0}\right)$ days, $\tau_{C S M_{\text {uniform }}}$ from Eq. 3 , and $m$ from $m=-\delta / 3$. With these assumptions, mass-loss rates, $\dot{M}$, can be calculated. 
From Eq. 9 and its modification for inclusion of a clumpy CSM (see the following Section) the mass-loss rates from SN progenitors are generally estimated to be $\sim 10^{-6} \mathrm{M}_{\odot} \mathrm{yr}^{-1}$ for Type Ib and Ic SNe and $\sim 10^{-4} \mathrm{M}_{\odot} \mathrm{yr}^{-1}$ for Type II SNe. For the specific case of SN1993J, where detailed radio observations are available starting only a few days after explosion, Van Dyk, et al. [20] find evidence for a changing mass-loss rate for the presupernova star which was as high as $\sim 10^{-4} \mathrm{M}_{\odot} \mathrm{yr}^{-1}$ approximately 1000 years before explosion and decreased to $\sim 10^{-5} \mathrm{M}_{\odot} \mathrm{yr}^{-1}$ just before explosion.

\section{Clumpiness of the Presupernova Wind}

In their study of the radio emission from SN1986J, Weiler, Panagia, \& Sramek [5] found that the simple Chevalier [6,7] model could not describe the relatively slow turn-on. They therefore added terms described mathematically by $\tau_{\mathrm{CSM}_{\text {clumps }}}$ in Eqs. 1 and 5. This extension greatly improved the quality of the fit and was interpreted by [5] to represent the possible presence of filamentation or clumpiness in the CSM. Such a clumpiness in the wind material was again required for modeling the radio data from SN1988Z [21] and SN1993J [20]. Since that time, evidence for filamentation in the envelopes of SNe has also been found from optical and UV observations (see, e.g., $[22,23]$ ).

\section{Binary Systems}

In the process of analyzing a full decade of radio measurements from SN1979C, $[17,18]$ found evidence for a significant, quasi-periodic, variation in the amplitude of the radio emission at all wavelengths of $\sim 15 \%$ with a period of 1575 days or $\sim 4.3$ years (see Fig. 3a at age $<4000$ days). They interpreted the variation as due to a minor $(\sim 8 \%)$ density modulation, with a period of $\sim 4000$ years, on the larger, relatively constant presupernova stellar mass-loss rate. Since such a long period is inconsistent with most models for stellar pulsations, they concluded that the modulation may be produced by interaction of a binary companion in an eccentric orbit with the stellar wind from the presupernova star.

This concept was strengthened by more detailed calculations for a binary model from Schwarz \& Pringle [19]. Since that time, the presence of binary companions has been suggested for the progenitors of SN1993J [24] and SN1994I [25], indicating that binaries may be common in presupernova systems.

\section{HII Along the Line-of-Sight}

A reanalysis of the radio data for SN1978K from Ryder, et al. [26] clearly shows flux density evolution characteristic of normal Type II SNe. Additionally, the data indicate the need for a time-independent, free-free absorption component. Montes, 
et al. [9] interpret this constant absorption term as indicative of the presence of HII along the line-of-sight to SN1978K, perhaps as part of an HII region or a distant circumstellar shell associated with the SN progenitor. SN1978K had already been noted by [26] for its lack of optical emission lines broader than a few thousand $\mathrm{km} \mathrm{s}^{-1}$ since its discovery in 1990, indeed suggesting the presence of slowly moving circumstellar material.

To determine the nature of this absorbing region, a high-dispersion spectrum of SN1978K at the wavelength range $6530-6610 \AA$ was obtained by Chu, et al. [27]. The spectrum shows not only the moderately broad $\mathrm{H} \alpha$ emission of the SN ejecta, but also narrow nebular $\mathrm{H} \alpha$ and [N II] emission. The high [N II] $6583 / \mathrm{H} \alpha$ ratio of $0.8-1.3$ suggests that this radio absorbing region is a stellar ejecta nebula.

\section{Rapid Presupernova Stellar Evolution}

SN radio emission that preserves its spectral index while deviating from the standard model is taken to be evidence for a change of the average circumstellar density behavior from the canonical $r^{-2}$ law expected for a presupernova wind with a constant mass-loss rate, $\dot{M}$, and a constant wind velocity, $w$. Since the radio luminosity of a $\mathrm{SN}$ is proportional to $(\dot{M} / w)^{(\gamma-7+12 m) / 4}$ [6] or, equivalently, to the same power of the circumstellar density (since $\rho_{\mathrm{CSM}} \propto M / w$ ), a measure of the deviation from the standard model provides an indication of deviation of the circumstellar density from the $r^{-2}$ law. Monitoring the radio light curves of RSNe also provides a rough estimate of the time scale of deviations in the presupernova stellar wind density. Since the SN blastwave travels through the CSM roughly 1,000 times faster than the stellar wind velocity which established the CSM ( $v_{\text {blastwave }} \sim$ $10,000 \mathrm{~km} \mathrm{~s}^{-1}$ vs. $w_{\text {wind }} \sim 10 \mathrm{~km} \mathrm{~s}^{-1}$ ), one year of radio light curve monitoring samples roughly 1000 years of stellar wind evolution.

\section{Sphericity of an SN Explosion}

It has often been suggested that $\mathrm{SN}$ explosions are non-spherical, and there is evidence in a number of stellar systems for jets, lobes, and other directed massloss phenomena. Also, the presence of polarization in the optical light from SNe (including SN1993J) has been interpreted for non-sphericity (see, e.g., [28]) and probably the most obvious evidence for non-spherical structure in an SN system is the very prominent inner ring around SN1987A. However, our most direct evidence for the structure of at least the blastwave from an SN explosion and the CSM with which it is interacting is from VLBI measurements on SN1993J. A series of images taken by Marcaide, et al. [1] over a period of two years from 1994 September through 1996 October show only a very regular ring shape indicative of a relatively spherical blastwave expanding into a relatively uniform CSM. The cause of such apparently conflicting results is still to be resolved. 


\section{Deceleration of Blastwave Expansion}

Radio studies also offer the only possibility for measuring the deceleration of the blastwave from the SN explosion. So far, this has been directly possible for two objects, SN1987A and SN1993J, although in some cases the deceleration can be estimated from model fitting to the radio light curves. Manchester, et al. [29] have shown that the blastwave from the explosion of SN1987A traveled through the tenuous medium of the bubble created by the high speed wind of its BSG progenitor at an average speed of $\sim 10 \%$ of the speed of light $\left(\sim 35,000 \mathrm{~km} \mathrm{~s}^{-1}\right)$, but has decelerated dramatically to only $\sim 3,000 \mathrm{~km} \mathrm{~s}^{-1}$ since it has reached the inner edge of the prominent optical ring.

Marcaide, et al. [1], through the use of VLBI techniques, have been able to follow the expansion of the radio shell of SN1993J in detail, and also find that it is starting to decelerate, although the deceleration is not nearly as dramatic as that seen for SN1987A. While the expansion speed of SN1993J is quite high at $\sim 15,000$ $\mathrm{km} \mathrm{s}^{-1}[1]$, the deceleration is much more gradual than that of SN1987A.

\section{Peak Radio Luminosities and Distances}

Long-term monitoring of the radio emission from SNe shows that the radio light curves evolve in a systematic fashion with a distinct peak flux density (and thus, in combination with a distance, a peak spectral luminosity) at each frequency and a well-defined time from explosion to that peak. Studying these two quantities at 6 $\mathrm{cm}$ wavelength, peak spectral luminosity $\left(L_{6 \mathrm{~cm}}\right.$ peak $)$ and time after explosion date $\left(t_{0}\right)$ to reach that peak $\left(t_{6 \mathrm{~cm} \text { peak }}-t_{0}\right)$, Weiler, et al. [3] find that they appear related. In particular, based on five objects, Type Ib and Ic SNe may be approximate radio standard candles with a peak $6 \mathrm{~cm}$ spectral luminosity of

$$
L_{6 \mathrm{~cm} \mathrm{peak}} \approx 1.3 \times 10^{27} \mathrm{erg} \mathrm{s}^{-1} \mathrm{~Hz}^{-1}
$$

and, based on 17 objects, Type II SNe appear to obey a relation

$$
L_{6 \mathrm{~cm} \text { peak }} \simeq 6.0 \times 10^{23}\left(t_{6 \mathrm{~cm} \text { peak }}-t_{0}\right)^{1.5} \mathrm{erg} \mathrm{s}^{-1} \mathrm{~Hz}^{-1}
$$

with time measured in days. (Note that the constants in Eqs. 10 and 11 are slightly different from those determined by [3] since there are now more objects available for inclusion.) If these relations are supported by further observations, they provide a means for determining distances to $\mathrm{SNe}$, and thus to their parent galaxies, from purely radio continuum observations.

KWW, \& MJM wish to thank the Office of Naval Research (ONR) for the 6.1 funding supporting this research. Additional information and data on radio emission from SNe can be found on http://rsd-www.nrl.navy.mil/7214/weiler/and linked pages. 


\section{REFERENCES}

1. Marcaide, J. M. et al., Astrophys. J. Lett. 486, L31 (1997)

2. Bartel, N. et al., Nature 318, 25 (1985)

3. Weiler, K. W., Van Dyk, S. D., Montes, M. J., Panagia, N., \& Sramek, R. A., Astrophys. J. 500, 51 (1998)

4. Weiler, K., Sramek, R., Panagia, N., van der Hulst, J., \& Salvati, M., Astrophys. J. 301, 790 (1986)

5. Weiler, K., Panagia, N., \& Sramek, R., Astrophys. J. 364, 611 (1990)

6. Chevalier, R. A., Astrophys. J. 259, 302 (1982)

7. Chevalier, R. A., Astrophys. J. Lett. 259, L85 (1982)

8. Chevalier, R. A., Astrophys. J. 499, 810 (1998)

9. Montes, M. J., Weiler, K. W., \& Panagia, N., Astrophys. J. 488, 792 (1997)

10. Natta, A. \& Panagia, N., Astrophys. J. 287, 228 (1984)

11. Lozinskaya, T. A., Supernovae and Stellar Wind in the Interstellar Medium, New York: AIP, 1992, p. 190

12. Chevalier, R. A. \& Fransson, C., Astrophys. J. 420, 268 (1994)

13. Sramek, R. A., Panagia, N. \& Weiler, K. W., Astrophys. J. Lett. 285, L59 (1984)

14. van Dyk, S. D., Sramek, R. A., Weiler, K. W. \& Panagia, N., Astrophys. J. 409, $162(1993)$

15. Montes, M. J., Weiler, K. W., Van Dyk, S. D., Panagia, N., Lacey, C. K., Sramek, R. A., \& Park, R., Astrophys. J. 532, 1124 (2000)

16. Montes, M. J., Van Dyk, S. D., Weiler, K. W., Sramek, R. A., \& Panagia, N., Astrophys. J. 506, 874 (1998)

17. Weiler, K., Van Dyk, S., Panagia, N., Sramek, R., \& Discenna, J., Astrophys. J. 380, 161 (1991)

18. Weiler, K., Van Dyk, S., Pringle, J., \& Panagia, N., Astrophys. J. 399, 672 (1992)

19. Schwarz, D. H. \& Pringle, J. E., Mon. Not. R. Astr. Soc. 282, 1018 (1996)

20. Van Dyk, S., Weiler, K., Sramek, R., Rupen, M., \& Panagia, N., Astrophys. J. Lett. 432, L115 (1994)

21. Van Dyk, S., Sramek, R. A., Weiler, K., \& Panagia, N., Astrophys. J. Lett. 419, L69 (1993)

22. Filippenko, A., Matheson, T., \& Barth, A., Astron. J. 108, 222 (1994)

23. Spyromilio, J., Mon. Not. R. Astr. Soc. 266, 61 (1994)

24. Podsiadlowski, Ph., Hsu, J., Joss, P., \& Ross, R., Nature 364, 509 (1993)

25. Nomoto, K., Yamaoka, H., Pols, O. R., van den Heuvel, E., Iwamoto, K., Kumagai, S., \& Shigeyama, T., Nature 371, 227 (1994)

26. Ryder, S., Staveley-Smith, L., Dopita, M., Petre, R., Colbert, E., Malin, D., \& Schlegel, E., Astrophys. J. 417, 167 (1993)

27. Chu, Y.-H., Caulet, A., Montes, M. J., Panagia, N., Van Dyk, S. D., \& Weiler, K. W., Astrophys. J. Lett. 512, L51 (1999)

28. Hoeflich, P., Wheeler, J. C., Hines, D. C., \& Tramaell, S. R., Astrophys. J. 459, 307 (1996)

29. Manchester, R., Gaensler, B., Wheaton, V., Staveley-Smith, L., Tzioumis, A., Kesteven, M., Reynolds, J., \& Bizunok, N., Pub. Ast. Soc. Aust., in prep. (2001) 